TITLE:

\title{
Interfacial properties of polystyrene thin films as revealed by neutron reflectivity
}

\section{$\operatorname{AUTHOR(S):~}$}

Inoue, Rintaro; Kawashima, Kazuko; Matsui, Kazuya; Nakamura, Makoto; Nishida, Koji; Kanaya, Toshiji; Yamada, Norifumi

\section{CITATION:}

Inoue, Rintaro ... [et al]. Interfacial properties of polystyrene thin films as revealed by neutron reflectivity. Physical Review E 2011, 84(3): 031802.

\author{
ISSUE DATE: \\ 2011-09 \\ URL: \\ http://hdl.handle.net/2433/148007 \\ RIGHT: \\ (C2011 American Physical Society
}


PHYSICAL REVIEW E 84, 031802 (2011)

\title{
Interfacial properties of polystyrene thin films as revealed by neutron reflectivity
}

\author{
Rintaro Inoue, ${ }^{1, *}$ Kazuko Kawashima, ${ }^{1}$ Kazuya Matsui, ${ }^{1}$ Makoto Nakamura, ${ }^{1}$ Koji Nishida, ${ }^{1}$ \\ Toshiji Kanaya, ${ }^{1, \dagger}$ and Norifumi L. Yamada ${ }^{2}$ \\ ${ }^{1}$ Institute for Chemical Research, Kyoto University, Uji, Kyoto-fu 611-0011, Japan \\ ${ }^{2}$ High Energy Accelerator Research Organization, Tsukuba, Ibaraki-ken 305-0801, Japan
}

(Received 18 April 2011; published 16 September 2011)

\begin{abstract}
We have studied the glass transition temperature $\left(T_{g}\right)$ and molecular mobility of polystyrene (PS) thin films near the interface between the polymer thin film and substrate with bilayer thin films consisting of surface hydrogenated PS (h-PS) and bottom deuterated PS (d-PS) using neutron reflectivity. With decreasing the thickness of the bottom d-PS layer, $T_{g}$ near the interface between the polymer thin film and substrate increased compared to bulk $T_{g}$ and a drastic increase of $T_{g}$ was observed for the bottom d-PS layer $<155 \AA$ thick. The orientation of polymer chains at the interface is supposed to be related to the increase of $T_{g}$ near the interface between the polymer and substrate. The polymer chain mobility decreased with thickness even for the bottom d-PS layer with no discernible change of $T_{g}$. It is considered that the numerous contacts between polymer chains and substrate are related to the decrease of mobility near the interface between the polymer thin film and substrate.
\end{abstract}

DOI: 10.1103/PhysRevE.84.031802

PACS number(s): 61.41.+e, 64.70.pj, 68.60.Dv

\section{INTRODUCTION}

Understanding the mechanical and thermal properties of polymer thin films is crucial for industrial applications such as adhesives, lubricants, coating, and so on. Especially, the glass transition temperature $\left(T_{g}\right)$ of polymer thin films is so far extensively studied by various methods including reflectivity method [1-3], scanning probe microscope (SPM) [4-6], optical birefringence measurements [7], dielectric relaxation [8-10], and ellipsometry $[11,12]$ because $T_{g}$ is directly related to the thermal stability of polymer thin films. After the systematic studies on the thickness dependence of $T_{g}$ on polystyrene (PS) thin films supported on Si substrate [11], it was widely recognized that $T_{g}$ decreases with thickness for PS thin films and a reduction of $T_{g}$ with thickness was understood in terms of a mobile surface layer $[4,6]$. On the other hand, an increase of $T_{g}$ with decreasing thickness was observed for poly(methyl methacrylate) (PMMA) thin films supported on Si substrate with native oxide [11], implying a strong interfacial interaction between PMMA thin film and substrate. Different physical properties of the free surface or the interfacial region between polymer thin film and substrate seems to be related for the anomalous glass transition of the polymer thin film, hence we have to reveal the heterogeneous dynamics of polymer thin films along the depth direction to understand the anomalous physical properties of polymer thin films. In our former works, we studied the distributions of $T_{g}$ and thermal expansivity in PS thin films consisting of alternately stacked deuterated PS (d-PS) and hydrogenated PS (h-PS) layers by neutron reflectivity (NR) $[13,14]$ and observed a continuous distribution of $T_{g}$ from the free surface to the interface between substrate and polymer thin film. Interestingly only the bottom layer exhibited a quite small thermal expansivity and high $T_{g}$, which was too high to detect in the experimental temperature range. It is considered that the anomalous physical property of the bottom layer might be related to a kind of interaction

\footnotetext{
*rintaro@scl.kyoto-u.ac.jp

†kanaya@scl.kyoto-u.ac.jp
}

between the polymer thin film and substrate. Due to the experimental difficulty, the approaches for the evaluation of $T_{g}$ near the interface between polymer and substrate in polymer thin films were quite limited including both direct $[15,16]$ and indirect methods $[10,17,18]$. As far as the authors know, the direct evaluation of $T_{g}$ near the interface between polymer and substrate in polymer thin films has been so far performed by Tanaka and co-workers [15] and the group of Ellison and Torkelson [16] only. Tanaka et al. studied $T_{g}$ near the interface between PS thin film and substrate with PS labeled by dye molecules using time- and space-resolved fluorescence spectroscopy and reported the dramatic increase of $T_{g}$ with approaching the substrate [15]; on the other hand Ellison and Torkelson did not observe a discernible change of $T_{g}$ compared to bulk $T_{g}$ at $120 \AA$ from the substrate [16]. The final agreement for $T_{g}$ near the interface between polymer thin film and substrate in polymer thin films is still missing up until now. We considered that the combination of isotope labeling and NR would be a powerful tool for the evaluation of $T_{g}$ near the interface between polymer thin film and substrate. Another advantage of NR is the direct evaluation of thickness and the interfacial roughness simultaneously; hence we can also study the molecular mobility from the temperature dependence of interfacial roughness under a fixed measurement time in addition to the evaluation of $T_{g}$.

In this paper, we study $T_{g}$ and the mobility of polymer thin film near the interface between polymer thin film and substrate with bilayer thin films consisting of the surface h-PS layer, which is thick enough to avoid the reduction of $T_{g}$, and the bottom d-PS layer with various thickness using NR.

\section{EXPERIMENTAL}

We used h-PS with molecular weight $\left(M_{w}\right)$ of $7.69 \times 10^{5}$ and molecular weight distributions $\left(M_{w} / M_{n}\right)$ of 1.18 and d-PS with molecular weight $\left(M_{w}\right)$ of $7.31 \times 10^{5}$ and molecular weight distributions $\left(M_{w} / M_{n}\right)$ of 1.08 . The bulk $T_{g}$ 's determined by differential scanning calorimetry (DSC) at the cooling rate of $1.5 \mathrm{~K} / \mathrm{min}$ were $376 \pm 2 \mathrm{~K}$ for both h-PS and 
d-PS, implying that both polymers had the same bulk $T_{g}$ within experimental error. The following procedures were used for the preparation of bilayer thin films. First we prepared a d-PS layer onto a Si substrate with a 3 -in. diameter by spin-coating toluene solutions at $2000 \mathrm{rpm}$ and then dried it in a vacuum oven at $343 \mathrm{~K}$ for $24 \mathrm{~h}$ after drying in a vacuum oven at room temperature for 2 days. For the preparation of the second layer, we used the water-floating method $[19,20]$. We immersed a Si wafer with a 4-in. diameter into piranha solution [14] and we prepared an h-PS layer onto a $\mathrm{Si}$ substrate with a 4-in. diameter by spin-coating toluene solutions. Such a prepared h-PS layer was transferred from the Si substrate with a 4-in. diameter to a water surface and collected onto the first d-PS layer, which had already been prepared on a Si substrate with a 3-in. diameter. We had to be concerned with the residual solvent in polymer thin films; some researchers have already studied this problem using chromatography [21], NR [22,23], and Fourier Transform Infrared (FT-IR) [23]. Especially, the results reported by Zhang et al. are the most reliable among them because they carefully studied this problem with the combination of FT-IR and NR covering the broad $Q$ range [23]. Referring to the experimental results by Zhang et al., no trace of toluene solvent was observed even for as-deposited PS thin film. It implies that we can safely ignore the residual solvent in polymer thin film for our system. Another point that we also have to consider is the surface modification of polymer thin film induced by water immersion to thin film. With regard to this problem, Seo and Satija carefully studied the interfacial structure at the interface between d-PS thin films and $\mathrm{D}_{2} \mathrm{O}$ by NR [24] and did not observe a depletion layer at the interface between thin film and water. Referring to the works by Zhang et al. and Seo and Satija, at least our drying process just described is enough for the elimination of residual solvent and water; hence we decided to dry the prepared bilayer film in the vacuum oven with the same drying process as previously described. For the evaluation of $T_{g}$ near the interface between polymer thin film and substrate, we have to select the proper thickness of the surface h-PS layer because the surface layer was responsible for the reduction of $T_{g}$ for PS thin film [1-12]. First we prepared bilayer thin films consisting of the bottom d-PS layer $\sim 900 \AA$ thick and the surface h-PS layer with various film thicknesses to evaluate $T_{g}$ of the surface h-PS layer. From the thickness dependence of $T_{g}$ of the surface h-PS layer, we can understand how much thickness of the surface h-PS layer was needed to observe the reduction of $T_{g}$. As the next step, we prepared bilayer thin films consisting of bottom d-PS layer with various thicknesses and surface h-PS layer with constant thickness for the evaluation of $T_{g}$ near the interface between polymer thin film and substrate. Prior to NR measurements, all the bilayer thin films were annealed at a temperature above bulk $T_{g}(=403 \mathrm{~K})$ for $5 \mathrm{~min}$ under vacuum to promote structural relaxation. We have to explain the reason for selecting this annealing condition. In our former works $[13,14]$, we studied the distributions of thermal expansivity and $T_{g}$ in thin films utilizing two sets of five-layered PS thin films with a component layer $\sim 200 \AA$ thick. To observe the annealing effect on five-layered PS thin films, we used different annealing conditions. One five-layered PS thin film was annealed at $353 \mathrm{~K}$ for $12 \mathrm{~h}$ and the other was annealed at $403 \mathrm{~K}$ for $5 \mathrm{~min}$. Negative thermal expansivities were observed for all the component layers of five-layered thin film annealed at $353 \mathrm{~K}$ for $12 \mathrm{~h}$. On the other hand, the negative thermal expansivities were not observed for the five-layered PS thin film annealed at $403 \mathrm{~K}$ for $5 \mathrm{~min}$. We used the same h-PS and d-PS as those used for five-layered PS thin film; the total film thickness of five-layered film ( $\sim 1000 \AA$ thick $)$ was similar to that of our bilayer thin films study. We considered that our annealing condition ( $403 \mathrm{~K}$ for $5 \mathrm{~min}$ ) would be applicable to bilayer thin films.

In addition to the annealing effect, we also have to care about interdiffusion between the surface h-PS layer and the bottom d-PS layer at the temperature above bulk $T_{g}$ because of small $\chi$ parameter values [25]. We calculated the time dependence of root mean square displacement (RMSD) of the center of mass of bulk PS to estimate the contribution from interdiffusion. For the calculation of RMDS, we used the bulk diffusion constant $(D)$ and shift factor reported by Karim et al. [26]. We calculated mean square displacement (MSD) at a temperature $T$ through the relation $\mathrm{MSD}=2 D(T) t$, where $D(T)$ and $t$ correspond to the diffusion constant at $T$ and measurement time $t$, respectively, under the assumption that the interdiffusion is isotropic. The contribution from interdiffusion was found to be $\sim 2 \AA$ under this annealing condition and we can safely neglect the contribution from interdiffusion by annealing. The expected RMSDs at $403 \mathrm{~K}$ and $413 \mathrm{~K}$ for $10 \mathrm{~min}$ are $9 \AA$ and $25 \AA$, respectively, and the contribution from interdiffusion is comparable to the thickness of the thinnest d-PS bottom layer, $\sim 55 \AA$ thick. For the reliable determination of thickness of layer the contribution from interdiffusion must not exceed the thickness of the component layer, hence we just limited the measurement time to 10 min under the assumption that interdiffusion in thin film was the same as bulk. NR measurements were performed with ARISA-II reflectometer [27] installed at BL16 in J-PARC, Tokai.

\section{RESULTS AND DISCUSSION}

Figure 1 shows the observed neutron reflectivity profiles from a bilayer thin film consisting of the bottom d-PS layer $\sim 880 \AA$ thick and the surface h-PS layer $\sim 1000 \AA$ thick, at temperatures below and above $T_{g}$. The clear fringes were observable even at above bulk $T_{g}$, implying that severe interdiffusion was avoided due to short measurement time. The density of PS thin films was the same as that of bulk within $0.5 \%$ experimental error reported by Wallace et al. [28]; hence we decided to use bulk scattering length density (SLD) for d-PS and h-PS to our fits. The bilayer model could describe the experimental results fairly well, supporting that no residual solvent remained in polymer thin film. The evaluated thickness of the surface h-PS layer as a function of temperature is shown in Fig. 2. The $T_{g}$ 's shown by solid arrows in Fig. 2 were evaluated from the linear least squares fit to both the glassy and the molten states, and the dotted and dashed lines were also drawn for the evaluation of error bars of $T_{g}$. The evaluated $T_{g}$ 's as a function of the thickness of surface h-PS layer are summarized in Fig. 3. We have not shown the results from the bottom d-PS layer $\sim 880 \AA$ thick; however; the evaluated $T_{g}$ and thermal expansivity were the same as the bulk ones, implying that the bottom 


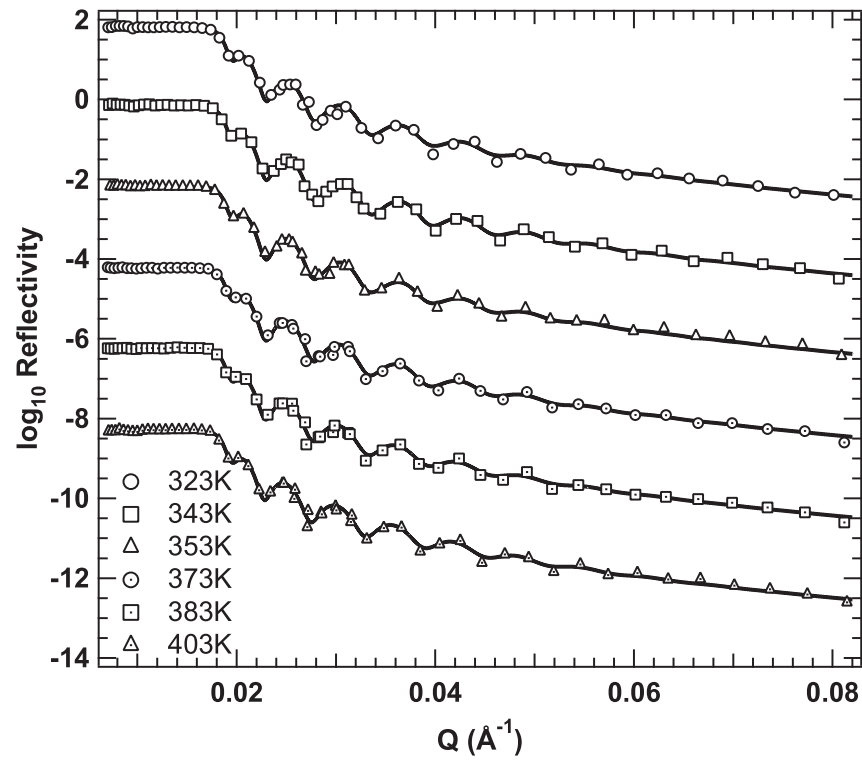

FIG. 1. Neutron reflectivity profiles from bilayer thin films consisting of surface h-PS layer $\sim 1000 \AA$ thick and bottom d-PS layer $\sim 880 \AA$, and fits using a bilayer model at several temperatures. For clarity, the reflectivity profiles and the corresponding fits were shifted vertically.

d-PS layer $\sim 880 \AA$ thick was bulklike as for $T_{g}$ and thermal expansivity. With decreasing the thickness of the surface h-PS layer, $T_{g}$ shifted to low temperature compared to bulk $T_{g}$ and the surface $T_{g}$ below $200 \AA$ thick leveled off to the constant value $(\sim 357 \mathrm{~K})$ within experimental error. Surface $T_{g}$ 's of PS thin films have already been studied by some researchers; we would like to check whether or not our evaluated surface $T_{g}$ was consistent with the former works. Fischer studied the surface $T_{g}$ of bulk PS by thermal probe atomic force microscope (AFM) [6] and reported that the surface $T_{g}$ and thickness of the surface layer was $\sim 356 \mathrm{~K}$ and $\sim 140 \AA$, respectively. From the optical birefringence measurements, Schwab et al. reported that $T_{g}$ at the surface region $\sim 70 \AA$ thick was $\sim 15-20 \mathrm{~K}$ lower than bulk $T_{g}$ [7]. Satomi et al. studied the surface region of PS thin films for several $M_{w}$ 's ranging from 4900 to 1450000 by SPM [5] and found that the surface $T_{g}$ was lower than bulk $T_{g}$ regardless of $M_{w}$ while the surface $T_{g}$ was dependent on $M_{w}$. Referring to the results of Satomi et al., the surface $T_{g}$ with similar $M_{w}$ used for our studies seemed to be around $350 \mathrm{~K}$ and this value is near to our evaluated surface $T_{\mathrm{g}}$ considering error bars. In our former work we have reported that $T_{g}$ of the surface layer $236 \AA$ thick in the five-layered thin film was $355 \pm 5 \mathrm{~K}$ [14]. Compared to the formally reported results, constant $T_{g}$ below $\sim 200 \AA$ thick is consistent with formerly reported results, supporting the validity of bilayer thin film for the selective determination of $T_{g}$ at the desired position along the depth direction. It was found that the surface h-PS layer thicker than $600 \AA$ was necessary for the determination of $T_{g}$ near the interface between polymer thin film and substrate from Fig. 3, therefore we selected the surface layer $\sim 880 \AA$ thick for our subsequent study.

The NR profiles from bilayer films consisting of the surface h-PS $\sim 880 \AA$ thick and the bottom d-PS layer with various

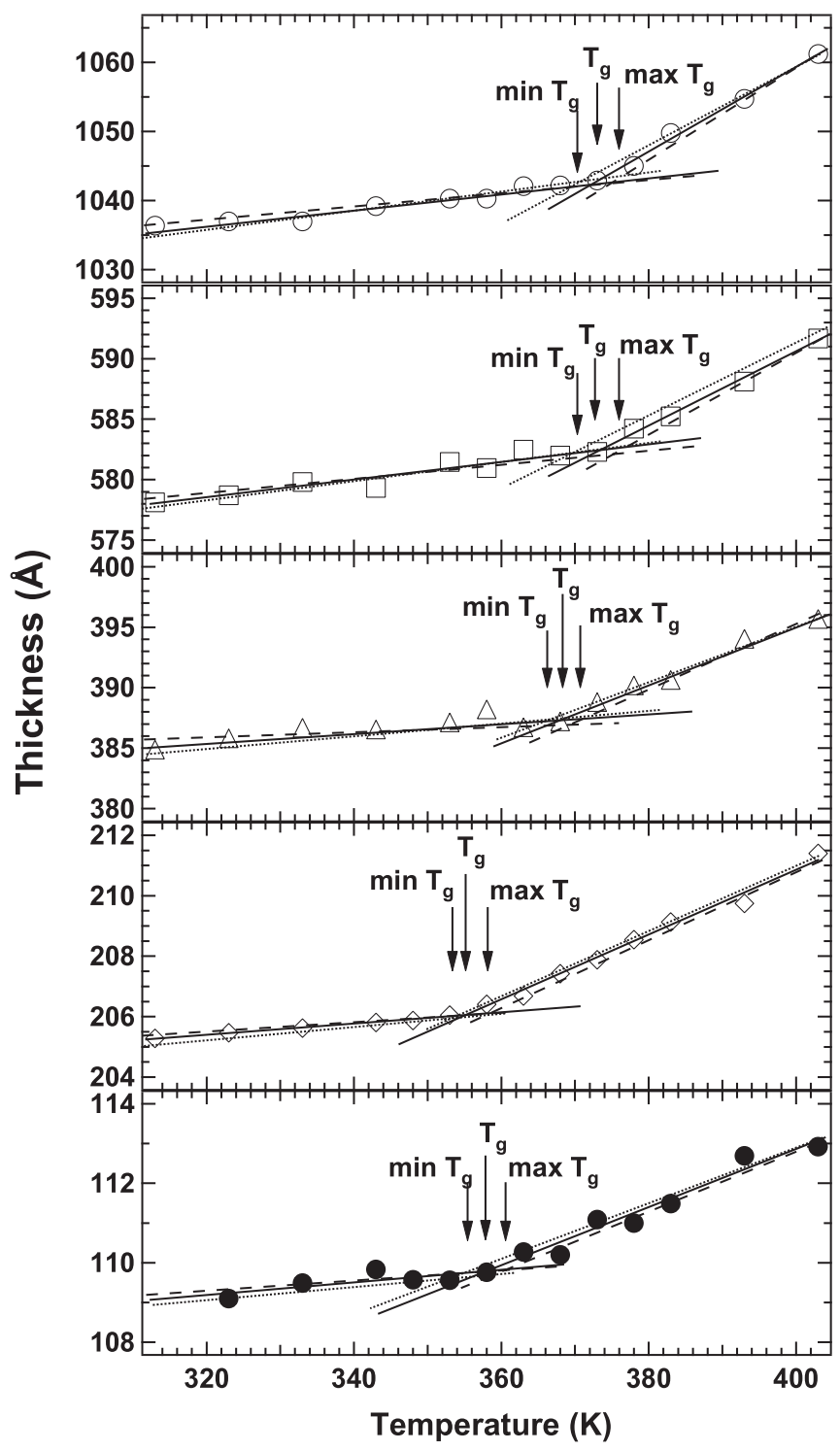

FIG. 2. Temperature dependence of thickness of the surface hPS layer; solid arrows correspond to the evaluated $T_{g}$ of surface h-PS layer with various thicknesses. Dotted and dashed lines are also included to evaluate the possible error bars for $T_{g}$.

film thicknesses at $313 \mathrm{~K}$ are shown in Fig. 4. By comparing the reflectivity profiles from bilayer thin films and a single d-PS layer whose thickness was equal to that of the bottom d-PS layer in bilayer film, it was found that the main fringes of reflectivity profiles from bilayer films were originated from d-PS layer. This is the main reason for the difference of reflectivity profiles from bilayer films consisting of the bottom d-PS with various thicknesses. The solid curves, which are the results of fits with the bilayer model could also describe the experimental results well. Evaluated $T_{g}$ 's from the surface h-PS layers were $373 \pm 3 \mathrm{~K}$ for all the bilayer thin films examined, indicating that the surface h-PS layer was thick enough to separate the surface effect. The temperature dependence of the thickness of bottom d-PS layer is shown in Fig. 5 and the clear change of thermal expansivity between the molten state and the glassy state was visible for the bottom d-PS layer thicker than $370 \AA$. The evaluated $T_{g}$ shown by 


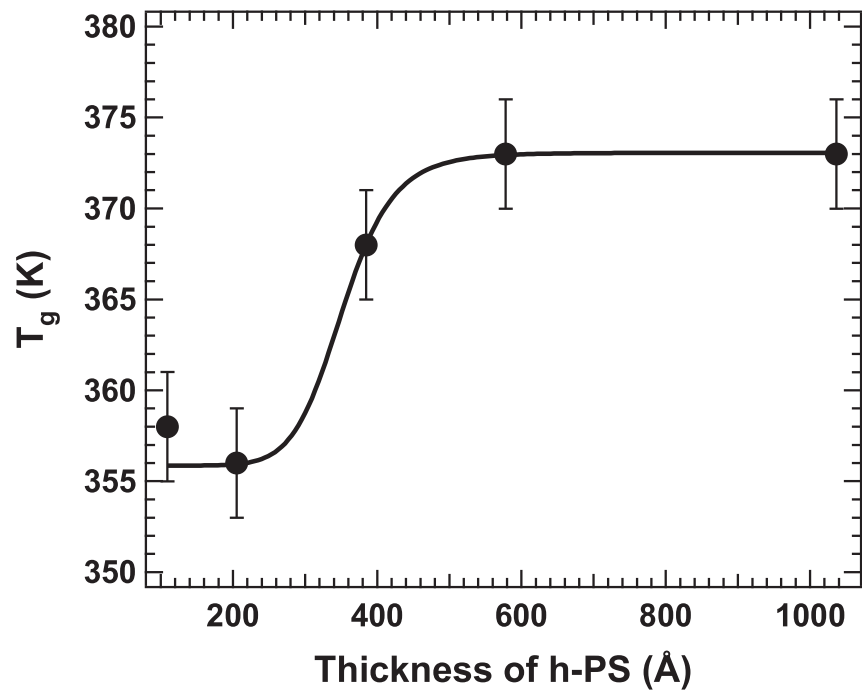

FIG. 3. The surface $T_{g}$ as a function of thickness of surface h-PS layer; the solid line was drawn by eye.

solid arrows shifted to high temperature as thickness was decreased, implying an onset of the interfacial effects between polymer thin film and substrate. For the bilayer film with the bottom d-PS layer $\sim 155 \AA$ thick, we observed zero or quite small thermal expansivity in the experimental temperature range examined. We have already observed the anomalous thermal expansivity for the bottom layer with similar thickness $(\sim 150 \AA)$ in five-layered thin films [14]. Tanaka et al. [15] observed an $\sim 20 \mathrm{~K}$ increase of $T_{g}$ compared to bulk $T_{g}$ at a distance $\sim 200 \AA$ away from the substrate for PS thin film supported to $\mathrm{SiO}_{x}$ substrate, whose surface property is similar to the $\mathrm{Si}$ substrate. The thickness of the bottom d-PS layer was $\sim 150 \AA$ below $200 \AA$, hence at least more than a 20 $\mathrm{K}$ increase of $T_{g}$ near the interface between polymer thin film

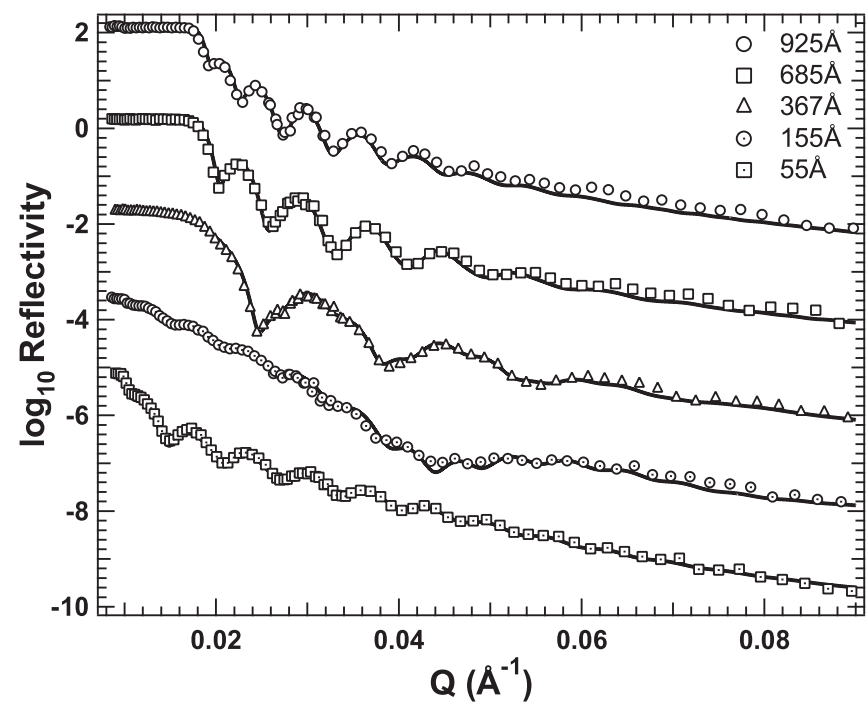

FIG. 4. Neutron reflectivity profiles from bilayer thin films consisting of surface h-PS layer $\sim 900 \AA$ thick and bottom d-PS layer with various thicknesses at $313 \mathrm{~K}$; solid lines were the results of fit using a bilayer model. The reflectivity profiles and the corresponding fits were shifted vertically for clarity.

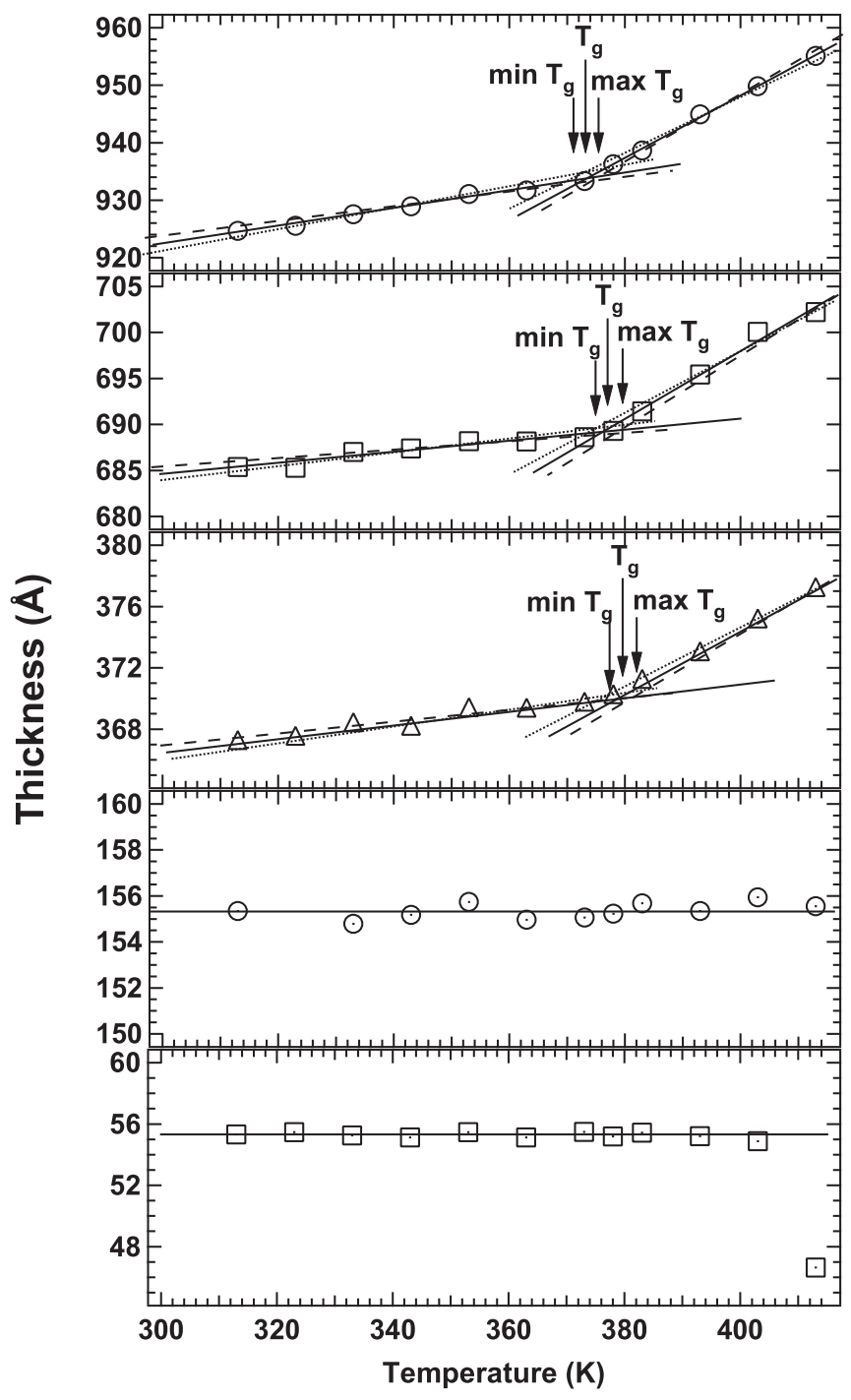

FIG. 5. Temperature dependence of thickness of bottom d-PS layer; solid arrows correspond to the evaluated $T_{g}$ 's of bottom layers with various thickness. Dotted and dashed lines are also included to evaluate the error bars for $T_{g}$.

and substrate would be expected. Considering the zero thermal expansivity and formerly reported result, $T_{g}$ shifted out of the experimentally accessible temperature range (above $413 \mathrm{~K}$ ) for the bottom d-PS layer $155 \AA$ thick. As previously described, $T_{g}$ of the surface h-PS layer in this bilayer thin film was the same as bulk $T_{g}$. In addition to the evaluation of surface $T_{g}$, we can also evaluate $T_{g}$ from the total thickness, and the evaluated $T_{g}$ was also the same as bulk $T_{g}$ despite the bottom d-PS layer. It was known that a PS thin film thicker than $1000 \AA$ was bulklike with regard to $T_{g}$ for PS thin films supported on Si substrate, implying that only the surface layer served as a detectable $T_{g}$ in the experimental temperature range. As for the bottom d-PS layer $\sim 55 \AA$ thick, thickness was almost independent of temperature up to $403 \mathrm{~K}$; however, the sudden reduction of thickness was observed at $413 \mathrm{~K}$. The reduction of film thickness with increasing temperature or so-called negative thermal expansivity was so far reported by some groups [1-3]. Orts et al. first observed the negative thermal expansivity for ultrathin PS films annealed at a temperature below bulk $T_{g}$ 
by X-ray reflectivity (XR) [3]. To confirm whether or not the annealing condition affects the resulting physical properties of polymer thin film, Miyazaki et al. systematically studied the annealing effect on PS thin films [29]. The negative thermal expansivity was observed for as-deposited thin film; on the other hand the negative thermal expansivity was not observed for PS thin films annealed at a temperature above bulk $T_{g}$; hence Miyazaki et al. concluded that the lack of annealing was responsible for the negative thermal expansivity observed for polymer thin film. One possibility for the negative thermal expansivity observed for the bottom d-PS $\sim 55 \AA$ thick is related to high $T_{g}$ near the interface between polymer thin film and substrate; therefore we tried to estimate $T_{g}$ for the bottom layer $\sim 55 \AA$ thick.

Referring to the works by Miyazaki et al. [29] carefully, film thickness increased with temperature in glassy state up to below bulk $T_{g}$ and began to decrease thickness at around bulk $T_{g}$ due to negative thermal expansivity. After the structural relaxation at above $T_{g}$ the film thickness started to increase with temperature due to the thermal expansion in the molten state. Their results implied the existence of two characteristic temperatures, at which film thickness decreased $\left(T_{d}\right)$ and thickness increased $\left(T_{i}\right)$ in the temperature range examined. It is supposed that real $T_{g}$ exists between $T_{d}$ and $T_{i}$; Miyazaki et al. found that $T_{g}$ for a well-annealed sample existed at the temperature between $T_{d}$ and $T_{i}$. We could not observe the temperature at which film thickness increased $\left(T_{i}\right)$ for the bottom layer $\sim 55 \AA$ thick; however, we could point out that $T_{g}$ of the bottom d-PS layer $\sim 55 \AA$ thick was higher than $413 \mathrm{~K}$. Recent coarse-grained molecular dynamics simulations [15] focusing on the evaluation of $T_{g}$ near the interface between polymer thin film and substrate supported the dramatic increase of $T_{g}$ below $100 \AA$ thick from the substrate, hence annealing at below $T_{g}$ seems to be related to the negative thermal expansivity observed for the bottom d-PS $55 \AA$ thick.

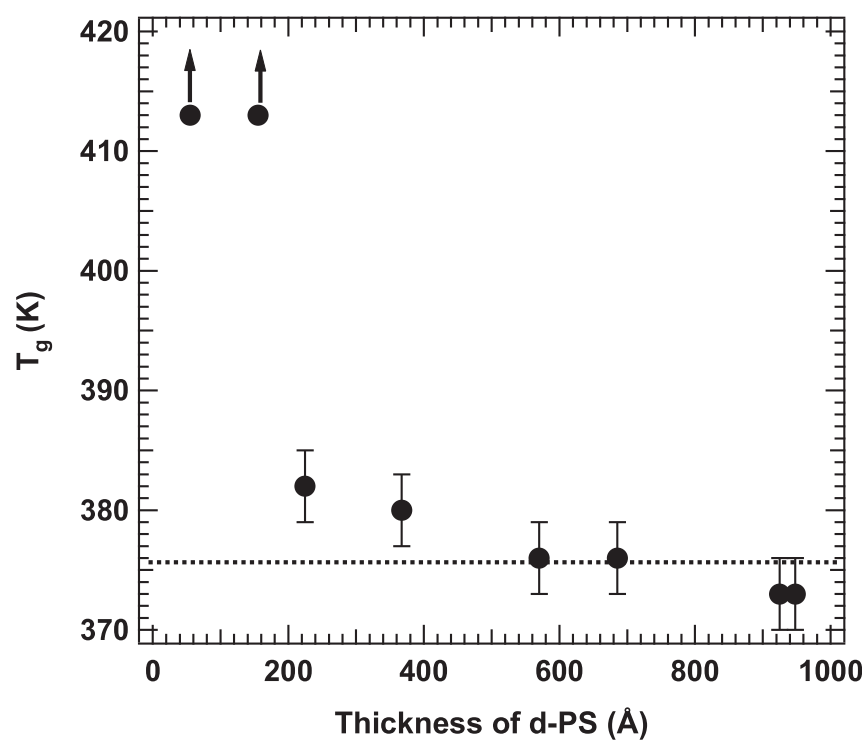

FIG. 6. Evaluated $T_{g}$ as a function of thickness of bottom d-PS layer, which is equal to the thickness from the substrate.
$T_{g}$ near the interface between polymer thin film and substrate as a function of the thickness of bottom d-PS layer was summarized in Fig. 6 and only the low limit was shown for the bottom d-PS layer $\sim 155 \AA$ and $\sim 55 \AA$ thick. We surely observed a dramatic increase of $T_{g}$ below the thickness of $200 \AA$ from the substrate and the onset of the interfacial effect was observed at $\sim 500 \AA$ from the substrate, considering the possible errors in the evaluated $T_{g}$. Although reported results for $T_{g}$ near the interface between polymer thin film and substrate are quite limited, we would like to compare the evaluated onset distance of the interfacial effect $(\sim 500 \AA)$ to the formerly reported results $[15,18]$. Tanaka et al. studied the depth dependence of $T_{g}$ near the interface between polymer thin film and substrate for three different inorganic substrates [15]. For $\mathrm{SiO}_{x}$ substrate, whose surface property is similar to the $\mathrm{Si}$ substrate, an increase of $T_{g}$ was observed at $\sim 550 \AA$ from the substrate. Wallace et al. reported that thickness and $T_{g}$ of the interfacial layer between polymer thin film and substrate was $\sim 400 \AA$ thick and higher than $433 \mathrm{~K}$, respectively, for PS supported by the hydrogen terminated Si substrate [18]. Our observed onset thickness for the interfacial effect $(\sim 500 \AA)$ seemed to be reasonable compared to the so-far reported results. We also would like to consider whether or not the interfacial thickness is related to the size of the polymer chain. For our studies, we used PS with $M_{w}$ of $\sim 700000$ to avoid interdiffusion between surface h-PS layer and bottom d-PS layer. $M_{w}$ 's of PS used for the works by Tanaka et al. [15] and Wallace et al. [18] were $M_{n}=53500$ and $M_{w}=233000$, respectively, implying that $R_{g}$ was not directly related to the onset thickness for the interfacial effect ( $\sim 500 \AA)$. Baschnagel and Binder suggested that the $R_{g}$ was not the largest scale characterizing the extent of the interfacial region from dynamic Monte Carlo studies [30]. Another simulation study [31] also showed that perturbations to the segment density due to wall effect persist if the distance is larger than $R_{g}$, supporting the long-range interfacial properties for polymer thin film.

So far some researchers tried to evaluate the averaged polymer chain conformation in the form of thin film by neutron scattering [32-34]. It is considered that the information concerning the polymer chain conformation near the interface between polymer thin film and substrate would offer a better clue to interpret our experimental results, which focused on selective evaluation of $T_{g}$ near the substrate instead of averaged polymer chain conformation. The evaluation of chain conformation near the substrate was performed with spectroscopy methods like sum-frequency spectroscopy (SFG) [35] and reflection-absorption IR (RAIR) [36]. Gautam et al. [35] studied the molecular orientation of PS thin films near the interface between polymer thin film and substrate with SFG spectroscopy and found that the polymer chains measured at room temperature were well oriented at the interface between polymer thin film and substrate. Furthermore, such oriented structure was unchanged even at $473 \mathrm{~K}$, which was $\sim 100 \mathrm{~K}$ above bulk $T_{g}$, suggesting that the oriented structure was hard to relax even at temperatures well above bulk $T_{g}$. With approaching the substrate the contribution from such oriented structure increases, resulting in the increase of $T_{g}$ near the interface between polymer and substrate. The universal thickness of the interfacial effect $(\sim 500 \AA)$ might correspond 
to the distance at which the oriented structure near the interface between polymer thin film and substrate dominates.

In order to obtain more detailed information regarding the interfacial property of polymer thin films, we also studied the temperature dependence of interfacial roughness between surface h-PS and d-PS. The interfacial roughness includes the contribution from initial roughness originating from the sample preparation and the interdiffusion between the surface h-PS layer and the bottom d-PS layer. As Stamm et al. reported, we could not neglect the contribution from initial roughness despite careful sample preparation [19,20]. To extract the interdiffusion contribution only, they subtracted the initial roughness from the interfacial roughness at a given time under the assumption that the initial roughness was not dependent on temperature and the temperature dependence of the roughness was attributed to the interdiffusion process. Following this procedure, Stamm et al. succeeded to monitor the different power laws of interfacial roughness for different time regimes [19] and their results were well explained within the framework of reptation theory. We also wanted to extract the contribution from interdiffusion only, hence we used $\Delta R$ by subtracting the roughness at room temperature from that at a given temperature. In principle $\Delta R$ is independent of the initial roughness or condition of the interfacial roughness; we can also discuss the molecular mobility near the interface between polymer thin film and substrate utilizing $\Delta R$ instead of interfacial roughness indirectly. Temperature dependence of $\Delta R$ is shown in Fig. 7. We also included the temperature dependence of root mean square displacement of bulk PS using the diffusion constant of center of mass of bulk PS as a solid line in Fig. 7 [26]. With a decrease in thickness of the bottom layer, we observed the decrease of $\Delta R$, implying the reduction of mobility with approaching the substrate. Interestingly the calculated bulk RMSD was larger than $\Delta R$ even for the bottom d-PS layer $\sim 1000 \AA$ thick, which was the same $T_{g}$ as bulk.

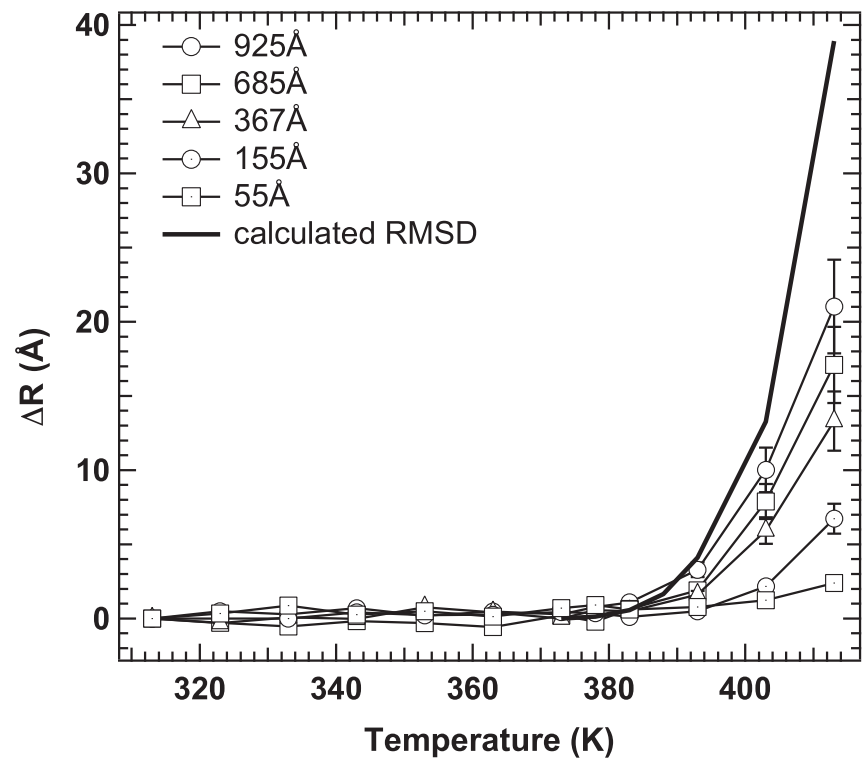

FIG. 7. Temperature dependence of $\Delta R$ between bottom d-PS layer and the surface h-PS layer as a function of thickness of bottom d-PS layer; solid line corresponds to the calculated root mean square displacement of the center of mass of bulk PS based on reptation.
Zheng et al. also studied the diffusion process of d-PS in matrix h-PS with different film thickness ranging from 1880 to $76 \AA$ by secondary ion mass spectroscopy (SIMS) [37] and found that the diffusion constant decreased even for the h-PS bottom layer with a thickness of $860 \AA$, which corresponded to $10 R_{g}$ of tracer d-PS. With a decrease in thickness of the bottom d-PS layer, further reduction of the diffusion constant was observed. We also observed the reduction of $\Delta R$ of the bottom d-PS layer $\sim 680 \AA$ thick compared to that of $\sim 890 \AA$ thick although the $T_{g}$ 's of the bottom layers were the same as bulk $T_{g}$. Such a slowing down of interdiffusion in polymer thin films compared to the dynamics of bulk was also reported by Kuhlman et al. [20] and Karim et al. [38], who monitored the interdiffusion at short times with multilayered thin film using NR. The slowing down of the diffusion constant compared to bulk seems to be a natural characteristic of polymer thin film. Zheng et al. discussed that the reduction of the diffusion constant was related to the shift of effective $T_{g}$ [37]. The advantage of our work is the direct evaluation of $T_{g}$ near the interface between polymer thin film and substrate. We have already known that an increase of $T_{g}$ was at $\sim 550 \AA$ from substrate, hence the reduction of molecular mobility for the bottom layer thicker than $550 \AA$ was not related to the change of $T_{g}$. Pu et al. studied the chain mobility of PS at the free surface by SIMS [39] and found that the diffusion coefficient was reduced compared to the bulk one within a distance of $4 R_{g}$ though $T_{g}$ near the free surface is lower than bulk $T_{g}$. It seems that $T_{g}$ is not necessary related to the reduction of molecular mobility; other factors might be concerned for the reduction of molecular mobility in the form of thin film. One possible reason for the reduction of molecular mobility is the increase of viscosity or entanglement density. Experimentally Barbero et al. reported that the effective viscosity reduced substantially for PS thin film $1000 \AA$ thick [40] and annealing at $T_{g}+55 \mathrm{~K}$ for $145 \mathrm{~h}$ was needed to recover the bulk viscosity from the reduced viscosity for PS thin film with $M_{w}=113000$ from the time dependence of the viscosity. Compared to the annealing condition performed by Barbero et al., our measurement time was too short to recover the bulk viscosity, hence the viscosity change in thin film cannot be used for a satisfactory explanation of the decrease of chain mobility near the interface between polymer thin film and substrate.

If the polymer chains were pinned at the substrate, there would exist numerous contacts between the substrate and polymer chains, resulting in the decrease of molecular mobility compared to that of bulk. The spectroscopic studies [35] revealed that the phenyl rings are oriented perpendicular to the surface normal at the interface between the substrate and polymer thin film, indicating the possibility of numerous contacts between polymer chains and substrate. Furthermore Fujii et al. reported the existence of bound or residual PS layer on the substrate even after rinsing thoroughly several times with toluene, which is a good solvent for PS [41], supporting the pinning or binding of polymer chains to the substrate.

\section{CONCLUSION}

In this paper, we have studied the $T_{g}$ and molecular mobility of polymer thin films near the interface between polymer thin film and substrate with bilayer thin films consisting of the 
surface h-PS layer with enough thickness to cap the surface effect and the bottom d-PS layer with various thicknesses using neutron reflectivity. With a decrease in thickness of the bottom d-PS layer, we observed an increase of $T_{g}$ compared to bulk $T_{g}$, implying the direct detection of an interfacial effect. The orientation of polymer chains at the interface is supposed to be related to the increase of $T_{g}$ near the interface between polymer and the substrate. The molecular mobility evaluated from the temperature dependence of the interfacial roughness between surface h-PS and bottom d-PS decreased compared to bulk RMSD even for the bottom d-PS layers with $T_{g}$, which were the same as bulk $T_{g}$. The numerous contacts or pinning of polymer chains to the substrate seemed to be related to the decrease of mobility near the interface polymer thin film and substrate.

\section{ACKNOWLEDGMENTS}

The neutron reflectivity experiments were performed in a Stype research project, High Energy Accelerator Organization (KEK) (Grant No. 2009S08).
[1] T. Miyazaki, K. Nishida, and T. Kanaya, Phys. Rev. E 69, 061803 (2004).

[2] T. Kanaya, T. Miyazaki, H. Watanabe, K. Nishida, H. Yamano, S. Tasaki, and D. B. Bucknall, Polymer 44, 3769 (2003).

[3] W. J. Orts, J. H. van Zanten, W.-l. Wu, and S. K. Satija, Phys. Rev. Lett. 71, 867 (1993).

[4] N. Satomi, A. Takahara, and T. Kajiyama, Macromolecules 32, 4474 (1999).

[5] N. Satomi, K. Tanaka, A. Takahara, T. Kajiyama, T. Ishizone, and S. Nakahama, Macromolecules 34, 8761 (2001).

[6] H. Fischer, Macromolecules 35, 3592 (2002).

[7] A. D. Schwab, D. G. Agra, J. H. Kim, S. Kumar, and A. Dhinojwala, Macromolecules 33, 4903 (2000).

[8] K. Fukao and Y. Miyamoto, Phys. Rev. E 61, 1743 (2000).

[9] A. Serghei, M. Tress, and F. Kremer, Macromolecules 39, 9385 (2006).

[10] A. Serghei, M. Tress, and F. Kremer, J. Chem. Phys. 131, 154904 (2009).

[11] J. L. Keddie, R. A. L. Jones, and R. A. Cory, Faraday Discuss. 98, 219 (1994).

[12] S. Kawana and R. A. L. Jones, Phys. Rev. E 63, 21501 (2001).

[13] T. Kanaya, R. Inoue, K. Kawashima, T. Miyazaki, I. Tsukushi, K. Shibata, G. Matsuba, K. Nishida, and M. Hino, J. Phys. Soc. Jpn. 78, 041004 (2009).

[14] R. Inoue, K. Kawashima, K. Matsui, T. Kanaya, K. Nishida, G. Matsuba, and M. Hino, Phys. Rev. E 83, 021801 (2011).

[15] K. Tanaka, Y. Tateishi, Y. Okada, T. Nagamura, M. Doi, and H. Morita, J. Phys. Chem. B 113, 4571 (2009).

[16] C. J. Ellison and J. M. Torkelson, Nat. Mater. 2, 695 (2003).

[17] K. Akabori, K. Tanaka, T. Nagamura, A. Takahara, and T. Kajiyama, Macromolecules 38, 9735 (2005).

[18] W. E. Wallace, J. H. van Zanten, and W. L. Wu, Phys. Rev. E 52, R3329 (1995).

[19] M. Stamm, S. Hüttenbach, G. Reiter, and T. Springer, Europhys. Lett. 14, 451 (1991).

[20] T. Kuhlmann, J. Kraus, P. Müller-Buschbaum, D. W. Schubert, and M. Stamm, J. Non-Cryst. Solids 235-237, 457 (1998).

[21] J. Gracía-Turiel and B. Jérome, Colloid. Polym. Sci. 285, 1617 (2007).
[22] J. Perlich, V. Korstgens, E. Metwalli, L. Schulz, R. Georgii, and P. Muller-Buchbaum, Macromolecules 42, 337 (2009).

[23] X. Zhang, K. G. Yager, S. Kang, N. J. Fredin, B. Akgun, S. Styija, J. F. Douglas, A. Karim, and R. L. Jones, Macromolecules 43, 1117 (2010).

[24] Y. Seo and S. Satija, Langmuir 22, 7113 (2006).

[25] F. S. Bates and G. D. Wignall, Phys. Rev. Lett. 57, 1429 (1986).

[26] A. Karim, A. Mansour, G. P. Felcher, and T. P. Russell, Phys. Rev. B 42, 6846 (1990).

[27] K. Mitamura, N. L. Yamada, H. Sagehashi, H. Seto, N. Torikai, T. Sugita, M. Furusaka, and A. Takahara, J. Phys.: Conf. Ser. 272, 012017 (2011).

[28] W. E. Wallace, N. C. Beck Tan, and W. L. Wu, J. Chem. Phys. 108, 3798 (1998).

[29] T. Miyazaki, K. Nishida, and T. Kanaya, Phys. Rev. E 69, 022801 (2004).

[30] J. Baschnagel and K. Binder, Macromolecules 28, 6808 (1995).

[31] I. Bitsanis and G. Hadziioannou, J. Chem. Phys. 92, 3827 (1990).

[32] R. L. Jones, S. K. Kumar, D. L. Ho, R. M. Briber, and T. P. Russell, Nature 400, 146 (1999).

[33] R. L. Jones, S. K. Kumar, D. L. Ho, R. M. Briber, and T. P. Russell, Macromolecules 34, 559 (2001).

[34] J. Kraus, P. Müller-Buschbaum, T. Kuhlmann, D. W. Schubert, and M. Stamm, Europhys. Lett. 49, 210 (2000).

[35] K. S. Gautam, A. D. Schwab, A. Dhinojwala, D. Zhang, S. M. Dougal, and M. S. Yeganeh, Phys. Rev. Lett. 85, 3854 (2000).

[36] J. M. Zhang, D. H. Zhang, and D. Y. Shen, Macromolecules 35, 5140 (2002).

[37] X. Zheng, M. H. Rafailovich, J. Sokolov, Y. Strzhemechny, S. A. Schwarz, B. B. Sauer, and M. Rubinstein, Phys. Rev. Lett. 79, 241 (1997).

[38] A. Karim, G. P. Felcher, and T. P. Russell, Macromolecules 27, 6973 (1994).

[39] Y. Pu, M. H. Rafailovich, J. Sokolov, D. Gersappe, T. Peterson, W.-L. Wu, and S. A. Schwarz, Phys. Rev. Lett. 87, 206101 (2001).

[40] D. R. Barbero and U. Steiner, Phys. Rev. Lett. 102, 248303 (2009).

[41] Y. Fujii, Z. Yang, J. Leach, H. Atarashi, K. Tanaka, and O. K. C. Tsui, Macromolecules 42, 7418 (2009). 